INRA Prod. Anim., 2005, 18 (3), 149-151

\title{
La petite histoire du phosphore en alimentation animale : les grandes étapes du demi-siècle*
}

L. GUEGUEN

INRA, Directeur de Recherches honoraire Courriel :leon.gueguen@free.fr

L'évolution de l'utilisation du phosphore (P) en alimentation animale peut être caractérisée en six grandes périodes depuis la seconde guerre mondiale. Ces périodes seront définies par les faits scientifiques ou les évènements marquants.

Avant 1955, la pénurie d'engrais phosphatés ayant sévi durant la guerre et les années d'après-guerre avait conduit à un net appauvrissement des fourrages en $\mathrm{P}$. La complémentation des rations était alors pourtant peu pratiquée, notamment chez les ruminants. Il en résultait des subcarences en $\mathrm{P}$ dont l'incidence était toutefois limitée chez les vaches laitières par le faible niveau des rendements. Le risque était bien moindre pour les monogastriques recevant des céréales et des farines animales riches en $\mathrm{P}$, le calcium étant alors le facteur limitant (rachitisme fréquent chez les porcs, coquilles d'huîtres broyées distribuées aux poules pondeuses). Le problème du phosphore était alors très préoccupant et avait justifié la tenue à Paris en 1949 de Journées du phosphore organisées par le CNRS. Les «normes» en vigueur étaient celles du NRC américain reprises partout en Europe et citées dans plusieurs revues publiées par des professeurs d'Ecoles vétérinaires (Ferrando, Jean-Blain) et de 1'Institut National Agronomique (INA) (A.-M. Leroy, J. Delage).

De 1955 à 1965, la prise de conscience de l'intérêt d'un apport supplémentaire de $\mathrm{P}$ a progressivement augmenté, notamment après la parution de mises au point de synthèse, l'une par J. Coléou en 1957 dans la Revue de l'élevage sur «Alimentation minérale des bovins», l'autre par Y. Henry en 1960 dans une brochure AFCA sur «les compléments dans l'alimentation animale». Je souhaite rendre ainsi hommage à ces deux collègues et amis récemment et trop tôt disparus. Dès lors, la complémentation minérale des rations s'est peu à peu organisée et généralisée, grâce à l'industrie de l'alimentation animale, mais est longtemps demeurée mal structurée et souvent fantaisiste. Elle s'imposait pourtant de plus en plus pour répondre à l'augmentation des performances zootechniques générée par le progrès génétique. Parallèlement, des travaux étaient réalisés à l'INRA de Jouy-en-Josas sur la composition minérale des fourrages, notamment sur les divers facteurs de variation des teneurs en phosphore (espèce, stade de développement, saison...) et une revue de synthèse était publiée sur la valeur comparée des phosphates minéraux, dénonçant les produits de mauvaise qualité. Enfin, les premiers travaux avec l'aide d'un traceur radioactif, ${ }^{32} \mathrm{P}$, étaient effectués à I'INRA de Jouy-en-Josas sur veau et mouton, dans le but d'évaluer la perte fécale endogène et l'absorption réelle de $\mathrm{P}$, première étape vers une estimation des besoins par la méthode factorielle.

De 1965 à 1973, le marché des phosphates a explosé pour diverses raisons : la publication des recommandations britanniques, relativement élevées, de l'Agricultural Research Council (ARC) obtenues par la méthode factorielle, en 1965 pour les Ruminants et en 1967 pour les Porcs, l'extension après 1970 du maïs ensilé pauvre en $\mathrm{P}$ chez les bovins, le remplacement partiel du maïs par du manioc très pauvre en $\mathrm{P}$ chez les monogastriques, les besoins accrus des animaux résultant de l'intensification de l'élevage. Il en est résulté divers travaux de recherche et expertises sur les critères de qualité des phosphates, notamment des essais comparatifs avec ${ }^{32} \mathrm{P}$ sur mouton et porc, et la «mise sur le marché» du test de solubilité citrique des phosphates, applicable aux composés minéraux et ayant au moins permis d'éradiquer les très mauvais phosphates.

La période 1973-1980 a d'abord été marquée par le choc pétrolier de 1973 avec ses conséquences plus ou moins directes : crise du soja (riche en P) et triplement du prix des phosphates marocains. Les phosphates sont donc subitement devenus très chers et des économies de $\mathrm{P}$ ont été encouragées, par exemple dans notre article paru en 1974 dans la revue «l'Elevage» sous le titre «Les phosphates sont chers : évitez donc les excès de phosphore!». Le retour prévisible des mauvais phosphates conduisit le Comité Interministériel de l'Industrie de l'Alimentation Animale (CIIAA) à valider en France en 1977 le test de solubilité citrique du phosphore. De nouvelles recommandations pour les ruminants furent publiées par l'INRA en 1978 et par l'ARC britannique en 1980 , ces dernières se distinguant par une forte baisse des besoins en phosphore des ruminants, principalement due à une évaluation faible du besoin net d'entretien. De nouvelles recommandations furent publiées aussi pour les porcs par l'INRA en 1979 (Guéguen et Perez 1979, 1981) et par l'ARC en 1981. Parallèlement, sévissait en France une théorie anti-phosphore, habilement propagée et basée sur le slogan «le phosphore est inutile et dangereux» (sans préoccupation environnementale à cette époque).

De 1980 à 1992, les travaux et mises au point scientifiques ont principale-

* Liste chronologique des principaux acteurs en recherche-développement sur le phosphore à l'INRA

Ruminants : L. Guéguen, J.-P. Bouchet, M. Durand, F. Meschy

Porcins : L. Guéguen, A. Pointillart, J.-M. Perez, J.-Y. Dourmad, C. Jondreville

Volailles : B. Sauveur, Y. Nys 
ment porté sur la réhabilitation du phosphore en nutrition des ruminants, d'une part pour confirmer la perte fécale endogène obligatoire chez le mouton et alimenter la controverse francobritannique sur le besoin d'entretien, d'autre part pour évaluer les besoins spécifiques en $\mathrm{P}$ soluble des microorganismes du rumen. Une journée du Cycle Approndi de 1'Alimentation Animale (CAAA) était organisée à l'INA Paris-Grignon en 1983 pour faire «le point sur le phosphore en alimentation animale», permettant en particulier de justifier la position de l'INRA sur le besoin d'entretien chez les ruminants et surtout de répondre aux détracteurs du phosphore. En 1984, l'INRA publiait de nouvelles recommandations pour les animaux monogastriques, avec notamment des recommandations d'apport de $\mathrm{P}$ pour les truies revues à la baisse (malgrè une polémique nationale). De 1986 à 1992, plusieurs mises au point successives sur la vache laitière, les bovins en croissance et la vache allaitante ont été publiées. En 1988, une mise à jour des recommandations de l'INRA pour les bovins, les ovins et les caprins était publiée et en 1991 paraissaient les nouvelles recommandations britanniques AFRC, marquées par un besoin d'entretien en hausse et variable en fonction de la quantité de matière sèche ingérée.

Après 1992, la préoccupation environnementale est apparue, avec le souci du rôle joué par le phosphore rejeté dans l'eutrophisation des eaux superficielles. Notre intervention au symposium Vitolmin-AFCA organisé à Angers en 1993 portait le titre «Bien nourrir les animaux en minéraux pour la santé de l'homme et son environnement». Cette période a aussi été marquée par le retour du blé plus riche en $\mathrm{P}$ et en phytase que le maïs et le manioc et surtout par l'introduction des phytases microbiennes dans l'alimentation des monogastriques. Le groupe «Phosphore» de la Commission Elevage du Corpen publiait en 1998 un rapport intitulé «Prévenir et réduire les risques de pollution des eaux par les phosphates provenant des activités agricoles»y. Enfin, en 2000, l'interdiction totale des farines de viande et d'os privait l'alimentation animale d'une quantité de P de bonne biodisponibilité et recyclable, soit un équivalent d'environ 100000 tonnes de phosphate bicalcique, accordant ainsi une nouvelle place aux phosphates minéraux.

Depuis une quinzaine d'années (et la cessation des programmes sur animaux d'élevage à l'INRA de Jouy-en-Josas), les travaux de recherche sur le phosphore ont été poursuivis, notamment sur le rôle et la biodisponibilité de $\mathrm{P}$ du rumen, l'activité phytasique des aliments, l'absorbabilité de $\mathrm{P}$, les rejets par les déjections, l'ajustement des apports aux besoins. Ils ont été principalement effectués à Paris-Grignon sur les ruminants, à Rennes-Saint-Gilles sur le porc et à Tours-Nouzilly sur les volailles. Depuis 2000, de nouveaux Apports Journaliers Recommandés (AJR) ont été publiés par le NRC américain (2001), la France (Meschy 2002) et les Pays-Bas (Valk et Beynen 2003). De nouveaux concepts ( $\mathrm{P}$ digestible chez le porc, $\mathrm{P}$ disponible chez les volailles, $\mathrm{P}$ absorbé chez les ruminants) ont été développés dans les Tables INRA-AFZ 2004 et présentés au cours des Journées AFTAA et AFZ (janvier 2004). Il est probable que de nouveaux ajustements seront effectués dans les prochaines années.

Il nous semble utile de revenir sur la définition et la signification de la notion d'AJR qui fait l'objet de révisions périodiques et, en l'occurrence, de révisions à la baisse pour le phosphore.

L'AJR, comme l'Apport Nutritionnel Conseillé (ANC) pour l'Homme, est un apport de sécurité qui vise à couvrir les besoins de la quasi-totalité d'une population. Il s'agit d'un concept basé sur des notions de probabilité et sur le principe suivant lequel, puisque le besoin de chaque individu n'est pas bien connu, l'apport qui doit être conseillé à chacun doit être assez élevé pour que les besoins de tous, notamment des individus les plus exigeants, aient le maximum de chances d'être couverts.

En prenant le modèle du calcium très bien étudié chez l'homme (l'apport de $\mathrm{P}$ étant toujours excédentaire), il est bien établi que le Besoin Nutritionnel Moyen (BNM), correspondant chez l'adulte à l'apport en dessous duquel le bilan calcique est négatif dans la moitié de la population, est de l'ordre de $700 \mathrm{mg} / \mathrm{j}$, avec un écart type de $100 \mathrm{mg} / \mathrm{j}$ (15\%). L'ANC (ou AJR) de $900 \mathrm{mg} / \mathrm{j}$ est obtenu en ajoutant deux écarts types au BNM, ce qui donne une probabilité de plus de $97 \%$ de ne pas être en bilan négatif. Cependant, cette probabilité tombe à $85 \%$ environ pour un apport de $800 \mathrm{mg} / \mathrm{j}$, à $50 \%$ pour un apport de $700 \mathrm{mg} / \mathrm{j}$ (BNM) et à $25 \%$ seulement pour un apport de $600 \mathrm{mg} / \mathrm{j}$. Si le BNM peut être adopté comme seuil d'inadéquation des apports, un apport de $800 \mathrm{mg} / \mathrm{j}(0,9 \mathrm{ANC})$ peut servir de valeur de référence pour définir la limite de risque acceptable.

Ce même raisonnement peut être appliqué aux AJR de phosphore chez les animaux, sans doute avec une souplesse plus grande que pour le calcium en nutrition humaine où toute erreur dans la prévention nutritionnelle de l'ostéoporose, notamment chez la Femme, doit être évitée. Si les AJR permettent, par définition, de couvrir avec le maximum de chances les besoins de tous les individus d'un troupeau, une plus grande probabilité de bilan négatif peut être acceptée, sans conséquence à moyen terme sur les performances des animaux à faible longévité, c'est-à-dire les volailles, les porcs à l'engrais et même les bovins en croissance. A noter que nos précédentes recommandations étaient assez généreuses pour les bovins en croissance et que, si l'on ne vise pas la minéralisation osseuse maximale (superflue dans la plupart des cas), une diminution des apports est possible. Le cas des vaches laitières fortes productrices est particulier car les besoins sont principalement constitués par le phosphore exporté par le lait, à un taux incompressible de $0,9 \mathrm{~g}$ par litre, et le risque d'insuffisance est alors moins acceptable.

Il importe aussi de noter que le coefficient de variation du besoin moyen, arbitrairement fixé à $15 \%$ chez l'homme, est certainement bien plus faible chez les animaux domestiques, 1'homogénéité génétique et physiologique d'un troupeau ou d'une bande étant considérablement plus grande que celle d'un groupe de la population. En conséquence, le besoin moyen et la limite basse de sécurité sont plus proches des AJR, ce qui exige un meilleur ajustement des apports aux besoins.

Quoi qu'il en soit, si le principe de précaution (ou ici de prévention) utilisé dans l'évaluation des AJR ne s'applique plus seulement à l'animal mais aussi, comme depuis une décennie, à l'environnement, il faut accepter une augmentation du risque individuel au profit d'une meilleure préservation de l'environnement. Il importe seulement que ce risque soit minimisé et nul doute que les récentes ré-évaluations, et les travaux de recherche à venir, parviendront au meilleur compromis. 


\section{Références principales}

AFRC, Agricultural and Food Research Council, 1991. A reappraisal of the calcium and phosphorus requirements of sheep and cattle. Nutr. Abstr. Rev., Ser. B, 61, 573-612.

ARC, Agricultural Research Council, 1965. The nutrient requirements of farm livestock, 2 . Ruminants. HMSO, London, UK.

ARC, Agricultural Research Council, 1967. The nutrient requirements of farm livestock, 3 . Pigs. HMSO, London, UK, 278p.

ARC, Agricultural Research Council, 1980 The nutrient requirements of ruminant livestock CAB. Slough. UK, 351p.

ARC, Agricultural Research Council, 1981. The nutrient requirements of pigs. CAB, Slough UK, 297p.

Guéguen L., Perez J.M., 1979. Recommended dietary allowances of minerals for pigs: situation in France. 30th Annual Meeting of the EAAP, Harrogate, England, 23-26 july, Commission on pig production, P1.3.

Guéguen L., Perez J.M., 1981. A re-evaluation of recommended dietary allowances of calcium and phosphorus for pigs. Proc. Nutr. Soc., 40, 273-278.

INRA, 1978. Alimentation des ruminants. R. Jarrige (ed), INRA Editions, Versailles, France, 621p.

INRA, 1984. L'Alimentation des animaux monogastriques : porc, lapin, volailles. INRA, Paris, France, 282p.

INRA, 1988. Alimentation des bovins, ovins et caprins. R. Jarrige (ed), INRA Editions, Versailles, France, $471 \mathrm{p}$.

INRA-AFZ, 2004. Tables de composition et de valeur nutritive des matières premières destinées aux animaux d'élevage : porcs, volailles, bovins, ovins, caprins, lapins, chevaux, poissons. D. Sauvant, J.-M. Perez, G. Tran (eds), $2^{\mathrm{e}}$ édition révisée, INRA, Paris, France, 301p.

Meschy F., 2002. Recommandations d'apport en phosphore absorbé chez les ruminants. Renc. Rech. Ruminants, Paris, France, 4-5 décembre 2002, 9, 279-285.

NRC, 2001. Nutrient requirements of dairy cattle. Tenth revised edition. National Academy Press, Washington DC, 381p.

Valk H., Beynen A.C., 2003. Proposal for the assessment of phosphorus requirements of dairy cows. Liv. Prod. Sci., 79, 267-272.

GUEGUEN L., 2005. La petite histoire du phosphore en alimentation animale : les grandes étapes du demi-siècle. INRA Prod. Anim., 18, 149-151. 
